\title{
UN CASO DE LECTURA DE PARAMENTOS Y ARGUMENTACIÓN CIENTÍFICA. S.PEDRO EL VIEJO DE ARLANZA, BURGOS-ESPAÑA
}

\author{
(ONE CASE OF "FACE READING" ANDITS SCIENTIFIC ARGUMENTATION
}

\author{
S. PEDRO EL VIEJO DE ARLANZA, BURGOS-SPAIN)
}

\author{
Luis Caballero Zoreda, Dr. Arqueólogo \\ Centro de Estudios Históricos-CSIC-Madrid \\ Leandro Cámara Muñoz, Arquitecto \\ Estudio de Arquitectura Latorre y Cámara-Madrid
}

ESPAÑA
Fecha de recepción: 13-П-95 $105-20$
RESUMEN

La pequeña ermita de S. Pedro el Viejo de Arlanza, situada junto al monasterio románico del mismo nombre, plantea problemas de adscripción cultural y cronológica, ya que, aunque sus caracteres son similares a los de la cercana iglesia de Quintanilla de las Viñas, considerada de época visigoda, las diferencias con el tipo constructivo visigodo hacian dudar de esta adscripción automática. Entre sus caracteres destaca el material, sillares romanos reutilizados, y una bóveda sobre pechinas que se dudaba si era coetánea a la primera construcción o pertenecía a la restauración barroca por la decoración que la recubria. El análisis de sus elementos estratigráficos ha permitido secuenciar cinco etapas en su construcción -prerrománica, románica, gótica, renacentista y barroca-, distinguir los elementos supervivientes de la primera construcción y reconstruir su forma primitiva. Pero, sobre todo, ha permitido plantear una nueva hipótesis sobre las hasta ahora consideradas "iglesias de época visigoda": la posibilidad de que en realidad sean mozárabes, construidas bajo influjo islámico entre los siglos VIII $y$ LX.

\section{SUMMARY}

The small hermit of S. Pedro el Viejo de Arlanza, located next to the Romanic monastery bearing the same name, poses problems of cultural and chronological assignment, since, in spite of the fact that it has similar characteristics as the nearby church of Quintanilla de las Viñas considered to belong to the Visigothic period, it presents some differences when compared to the Visigothic construction type. Some of its characteristics that particularly stand out are the material, the reused Roman ashlars, as well as a vault over panaches which might not be contemporary with the original construction, but may rather belong to the baroque restoration judging by the decoration it is covered with. The analysis of the stratigraphic elements allowed the sequencing into five construction stages, namely the pre-Romanic, Gothic, Renaissance and Baroque ones. This analysis also enabled the distinguishing of the elements remaining from the first construction and their reconstruction in the original form. Yet, what it permitted above all was the formulation of a new hypothesis on the so far considered "Visigothic churches", which consists of the possibility of these churches actually being Mozarabic, built under the Islamic influence between the VIII and LX centuries.

\section{Planteamiento del trabajo}

El método de lectura de paramentos se considera un eficaz instrumento para organizar la restauración de una construcción histórica, pero no debe olvidarse que básicamente es un instrumento de análisis histórico. El análisis de esta pequeña iglesia es un ejemplo de cómo este método sirve a la argumentación de una hipótesis histórica.
Con motivo de los trabajos realizados en el monasterio de S. Pedro de Arlanza, nuestro compañero Pablo Latorre nos llamó la atención sobre la ermita de S. Pedro el Viejo colocada en lo alto de un espolón aislado del valle del Arlanza, encima del monasterio románico. Una breve visita efectuada en el año 1990, hizo ver de inmediato su importancia, ya que tipológicamente podía considerarse de época visigoda por su sillería y la bóveda sobre pechinas de 


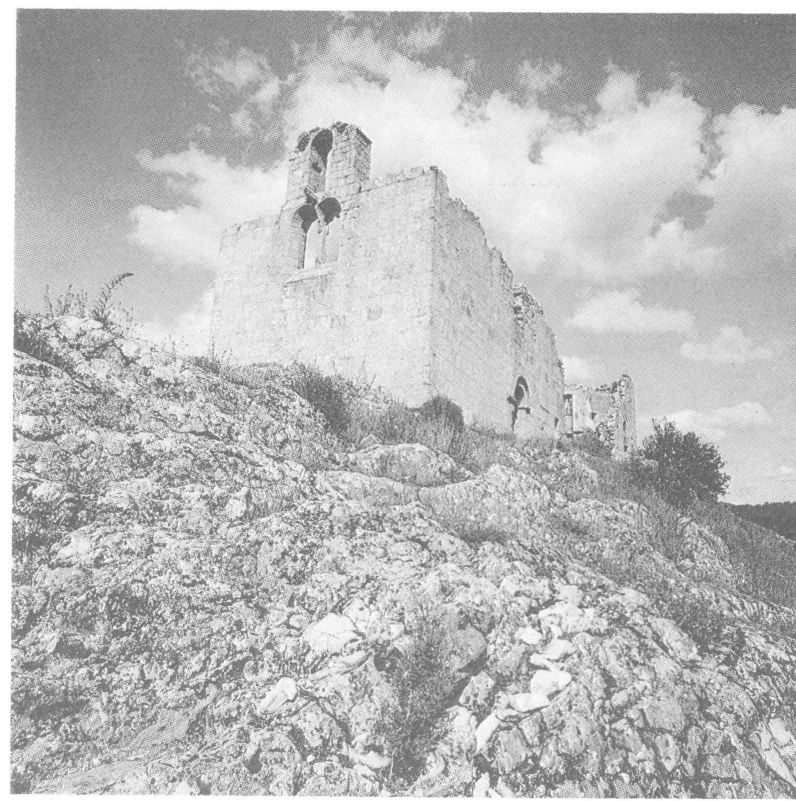

Fig.1.- La iglesia de S. Pedro el V'iejo de Arlanza desde S().

su ábside que recuerda las perdidas de la cercana iglesia de Quintanilla de las Viñas considerada de esa cronología. Por entonces Caballero creía encontrar contradicciones en parte de la argumentación tradicional sobre la arquitectura de nuestra alta Edad Media, en concreto la de época visigoda, y buscaba nuevos datos que ayudaran a confirmarla o a reformarla. Este edificio inédito suponía la posibilidad de contrastar esta argumentación, utilizando un método nuevo de análisis que, al separarse de los métodos tradicionales, facilitaría también el distanciamiento del paradigma consensuado.

Laermita de S. Pedrose había relacionado con el monasterio de S. Pedroy S. Pablo de Arlanza, citado documentalmente a partir del año 912, afirmando o rechazando que este monasterio prerrománico se encontrara en el lugar del actual románico, construido a partir de 1080 según una inscripción hoy perdida. De acuerdo con los esquemas científicos vigentes, la ermita podía ser la iglesia de un monasterio previo "de época visigoda", recuperado con la actividad "de Reconquista" en el s. X.

La ermita posee un aula rectangular de $14 \mathrm{~m}$ por $7,5 \mathrm{~m}$ y un ábside de planta casi cuadrada de $2,9 \times 3,4 \mathrm{~m}$, cubierta con la bóveda sobre pechinas citada. El edificio en 1990 se encontraba arruinado, con la cubierta del aula perdida y sólo restos de la última decoración barroca, encamonada y estucada, que había cubierto por completo su interior. Mientras que se conservó esta decoración apenas se consideró la posibilidad de que la bóveda del ábside fuera prerrománica ni por lo tanto la ermita, ya que tipológicamente tenía que ser barroca como parecía por su decoración. Sólo tras su ruina se pudo pensar en otras cronologías más antiguas, ya que además los aparejos y los elementos decorativos descubrían que el edificio había tenido otras etapas además de la prerrománica y la neoclásica. Muchos sillares - 23 entre los aún in situ y los removidos- eran inscripciones romanas reutilizadas y podía observarse que muchos más tenían huellas de gafas y grapas, dato significativo de su reutilización. También se observaban varios tipos de aparejos: de sillares grandes tallados con azuela, puntero y cincel; de sillares más pequeños tallados con gradina; de sillares de gradina con marcas; alternando distintos tipos de piedra; $y$, finalmente, de mampostería y adobe (Fig. 2). Además la ventana occidental y la puerta meridional podían catalogarse como tardogótico o renacentista. Era evidente que el edifício era adecuado para aplicar el análisis estratigráfico de paramentos, aunque fuera de modo experimental.

Con estos precedentes, las preguntas que se proponía contestar con este método eran las siguientes: cuántas etapas era posible distinguir en la ermita; si era posible diferenciar con seguridad la o las etapas prerrománicas y cuáles eran sus características, de modo que se pudiera decidir, por ejemplo, sobre la coetaneidad o no de bóveda y sillería; y qué indicios cronológicos -posiblemente relativos- podian descubrirse para fecharlas.

\section{Proceso de análisis}

Primero había que documentar gráficamente la ermita, lo que se inició con un sistema fotogramétrico analógico, pero su situación topográfica impidió la restitución completa, especialmente del exterior N. y S., por la inclinación del terreno, y del interior del ábside, por su pequeñez. Por ello la lectura se adelantó a la posesión de una adecuada documentación planimétrica, obligando a que los arquitectos del equipo, Cámara y Latorre, efectuaran croquis a mano alzada de todo el edificio (Figs. 3 y 9). Entretanto se había hecho operativo el equipo de fotogrametría analítico del Departamento de Historia Antigua y Arqueología del CEH, CSIC, más versátil, con el que se completó la planimetría que faltaba. Por lo tanto existían tres modelos distintos de documentación que se unificaron con el obtenido por el equipo del CSIC.

El análisis estratigráfico se desarrollóa pie de obra, a partir de 1991, sobre los croquis a mano alzada, distribuyéndose el trabajo en parejas de analistas. Para ello se dividió el edificio en cinco zonas -ábside y testeros E., N., S. y O. del aula-, aunque, ya iniciado el trabajo, su complicación obligó a replantear esta zonificación diferenciando como zona cada paramento del ábside, aunque se mantuvo la numeración de las zonas en las que ya se había iniciado el trabajo-1 a 7, consecutivamente muros S. del ábside, E. del aula y ábside, S. del aula, N. del ábside y O. y N. del aulaCada zona incluía un muro completo, tanto por fuera como por dentro, y a cada zona le correspondiān dos planos de alzado, exterior e interior, además de afgún detalle como las jambas de puertas y ventanas. 
La descripción y análisis propiamente arqueológico se realizó a partir de fichas diseñadas para el monumento, que resultaron demasiado teóricas y faltas de espacio para descripción y diagrama(Fig. 6). En la práctica su redacción fue demasiado prolija, usándose abundantes fichas anejas. El trabajo de campo duró unos quince días en total, trabajando un equipo de cuatro o cinco personas que rellenaron un total de 290 fichas. El mayor inconveniente procedió de dividir el trabajo en varias etapas con largas interrupciones entre sí, dejando para el final la realización de los diagramas. Ello dio lugar a dos problemas, uno de carácter más práctico, olvidar las relaciones existentes entre los elementos; y otrode carácter más teórico, cambios de orientación en la metodología derivados en gran parte de lo aprendido en otros trabajos.

Ante la falta de experiencia se decidió individualizar todos los elementos por el criterio de su continuidad física. La numeración fue aleatoria, tanto por zonas como por equipos, llevándose un listado con los números utilizados de modo que no se duplicaran. Se empezó por la cabecera y, de inmediato, el trabajo se complicó con las distintas y abundantes capas de estucos y enfoscados, en su mayoría barrocos. Se planteaban dos problemas, el de la multiplicación de elementos similares, con la misma forma $\mathrm{y}$ función, que en realidad pertenecían a una misma estructura; y el de enredarnos en un problema de relaciones que afectaban a una etapa moderna. Los mismos problemas se repetían en otros casos -huellas en sillares reutilizados, mechinales, trazas de la obra barroca, ...-. Se resolvieron añadiendo dos criterios de diferenciación más al ya usado, los de coetaneidad y funcionalidad constructiva. Por lo tanto en un momento avanzado del trabajo se decidió dar el mismo número a los elementos similares que, con absoluta seguridad, pertenecían a una misma y sincrónica acción constructiva. Como aún así seguían existiendo elementos similares individualizados, tanto por el proceso del trabajo como por el retraso que suponía unificar el criterio de similitud por parte de los equipos, al final del trabajo se completó la unificación renumerando todos los elementos tal como figuran definitivamente en el listado de elementos y en los planos. De este modo se redujeron a 141, número lógicamente mucho menor que el de elementos reales del edificio, al estar agrupados los similares bajo un mismo número, sea cual sea su situación topográfica. Así se simplifica también la comprensión del proceso y la numeración de los elementos se aproxima a su orden de deposición -los números bajos corresponden a elementos más antiguos y viceversa- y dentro de él a un orden topográfico. A pesar de que el estudio de estucosy enfoscados post-medievales (ss. XVI a XVIII) estaba muy avanzado, se englobaron como un solo elemento (E 95), apremiados por la falta de tiempo para individualizar en ellos subetapas cronológicas o procesos de trabajo.

Por otra parte, llegados a la fase final de reducción, se decidió no efectuar el paso de elementos a estructuras, lo
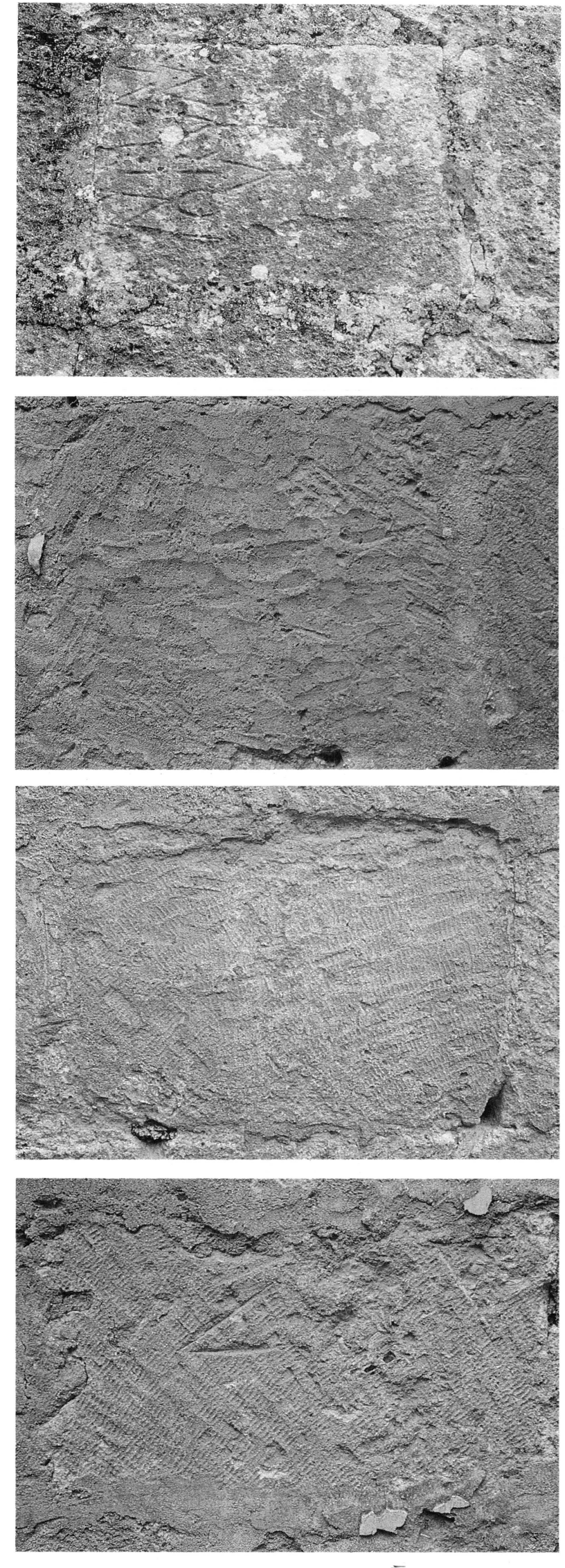

Fig.2.- Distintos tipos de técnica de labra de los sillares. A, estela romana reutilizaday comenzada a retallar. $B$, sillar romano reutilizado, retallado con azuela. $C$, sillar retallado con gradina. $D$, sillar retallado con gradina y con marca en forma de " $V "$. 


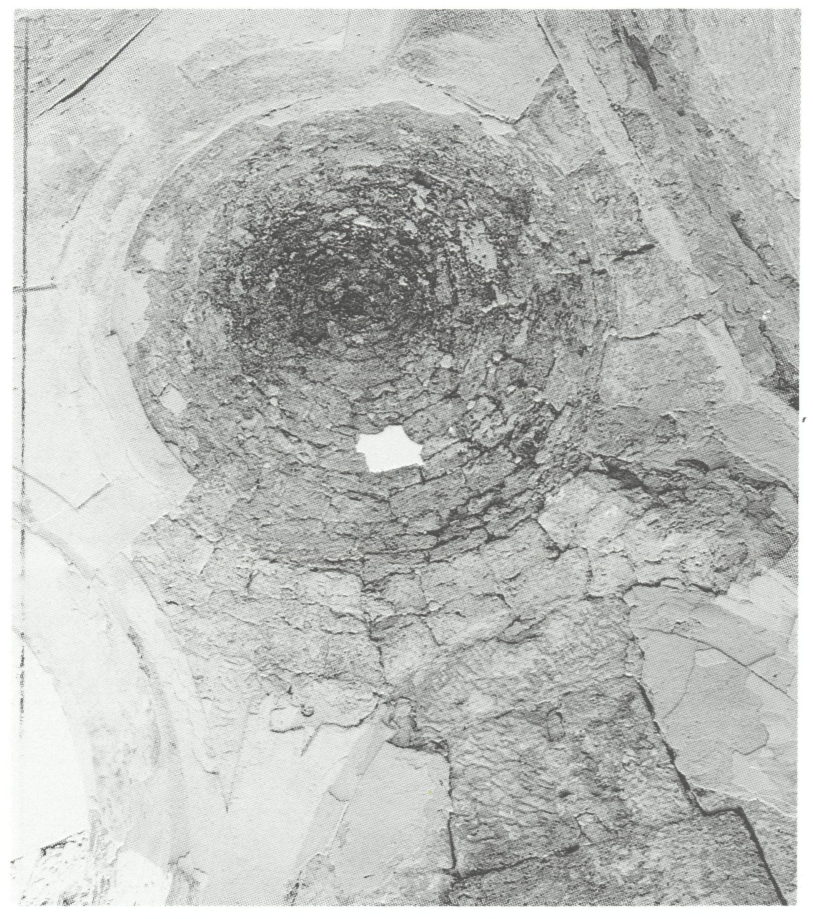

Fig.4.- La bóveda del ábside con sus tímpanos y pechinas. La sillería de muros ytimpanos es romana sin retallar; la bóveda, de sillarejo de piedra toba; en la cara N. se ha cortado la bóveda posiblemente para encajar un retablo; el estuco, de época barroca.

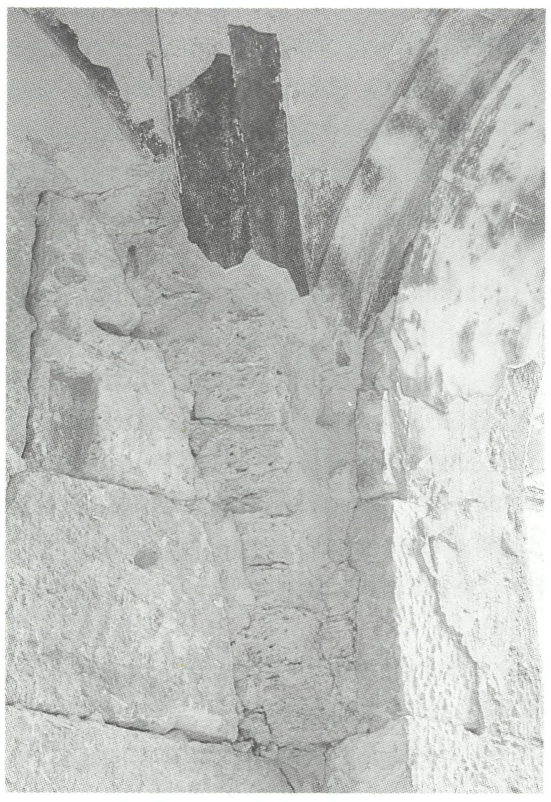

Fig.5.- Detalle del arranque de la pechina de la bóveda del ábside.

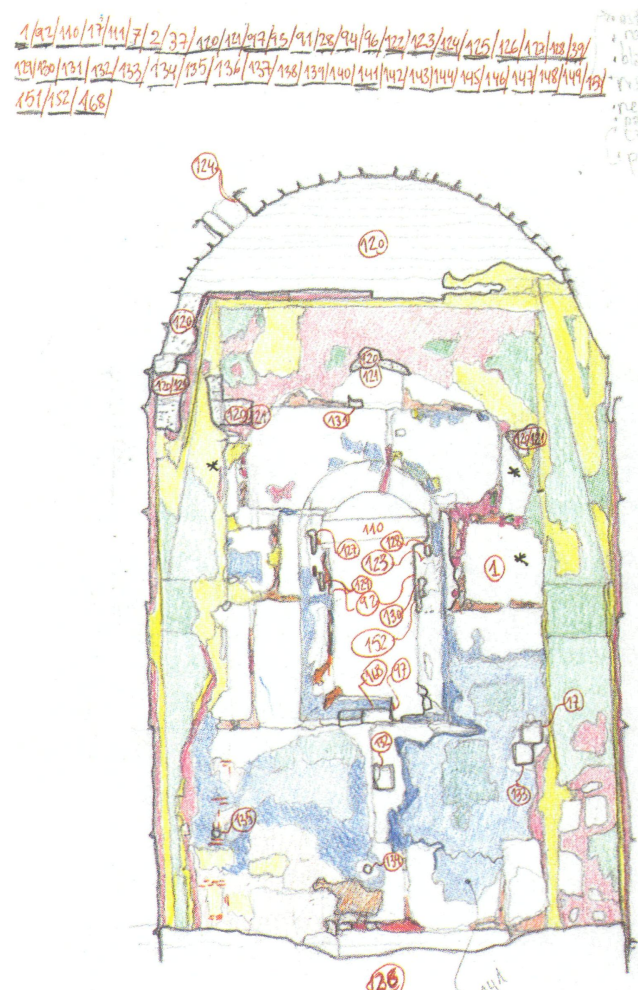

BONA 3, MURO ESTE ALSSI DE DXEDOR

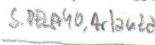

$12-13 \sqrt{u} \operatorname{ing} 91$

$16 \mathrm{~m}$

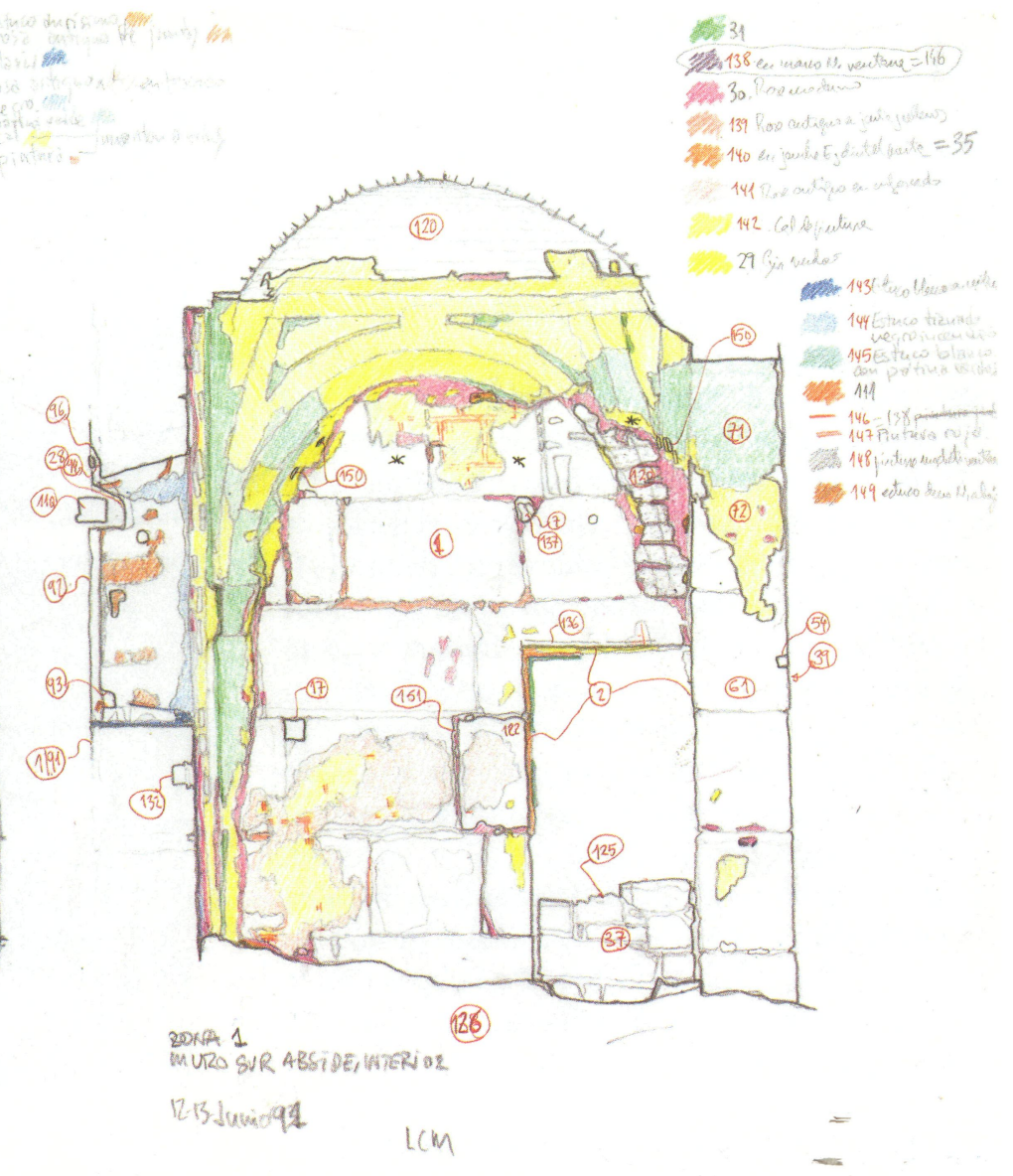

Fig.3.- Croquis de campo de las zonas 1 y 3, correspondientes a los muros $S$. y E. del ábside interior. 


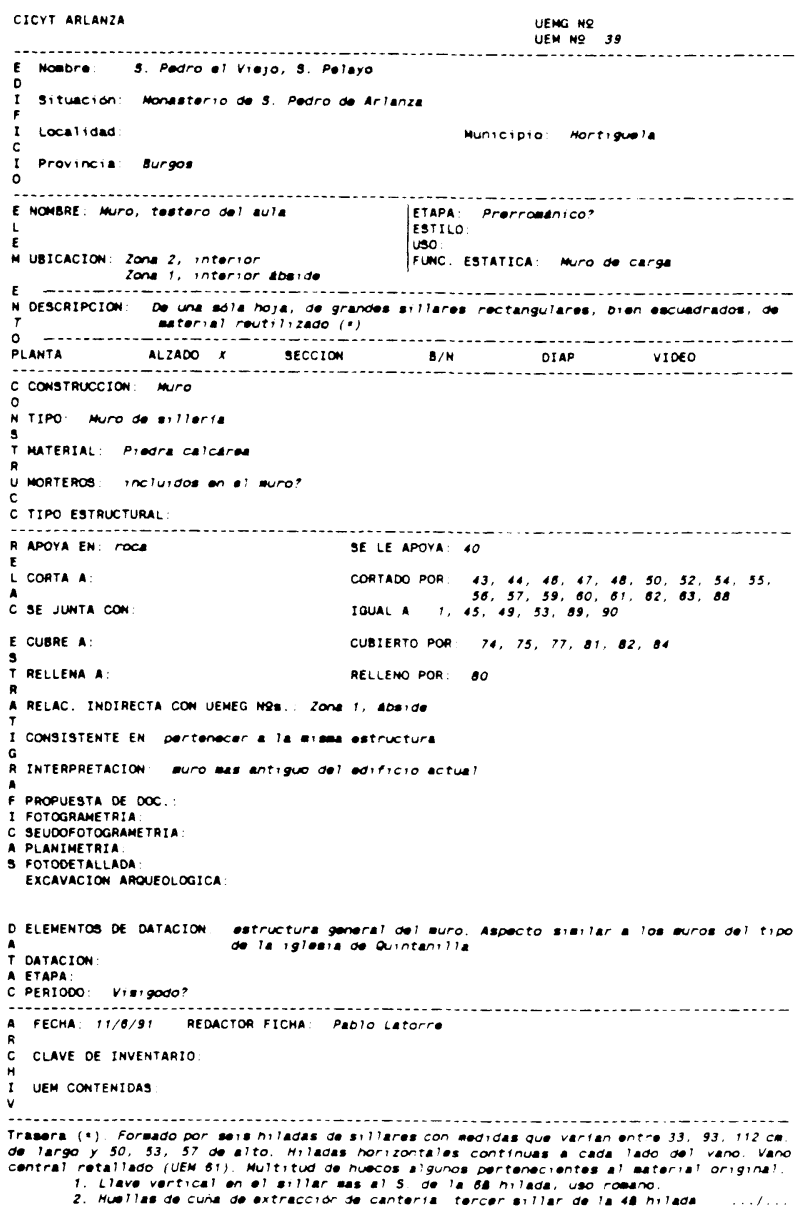

Fig.6.- Modelo de ficha utilizada en el trabajo de campo.

\begin{tabular}{|c|c|c|c|c|c|}
\hline$n$ & $\cos x \operatorname{xschsisin}$ & Aetroso & PImo & Postr:pi:a & minge:sen \\
\hline 1 & 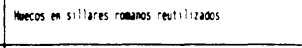 & $\circ$ & $2,3,5,9.10$ & & 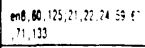 \\
\hline 2 & 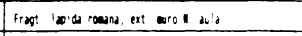 & 0 & 2 & & $\mathrm{eng}$ \\
\hline 3 & 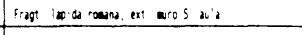 & 0 & 3 & & mon:2s \\
\hline 4 & 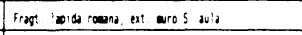 & 0 & 3 & & ans9 \\
\hline 3 & 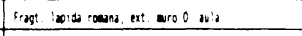 & $\theta$ & 5 & & en" \\
\hline 0 & frogt woun int wros wia & 0 & 1 & & mi2s \\
\hline & 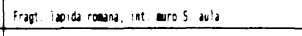 & 0 & $\dot{B}$ & & mi25 \\
\hline 1 & 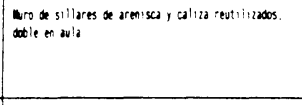 & ba & $2: 9$ & $+\infty x_{1}, 1,2$ & 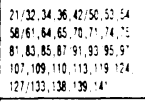 \\
\hline 9 & 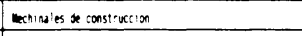 & 10 & 2,49 & & $27,2,2,37,46,01,32.32$ \\
\hline 10 & 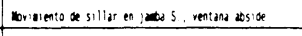 & is & 4.8 & & $20.8 .87,8,8,1,133,136$ \\
\hline " & 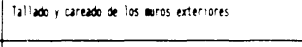 & 18 & 2is & & $\begin{array}{l}4.38 .879,106,224.32 \\
139\end{array}$ \\
\hline 18 & 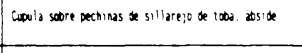 & $\therefore$ & 6.8 .1 & & 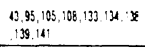 \\
\hline 13 & 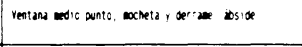 & !a & 0.68 & & 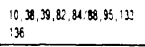 \\
\hline 14 & 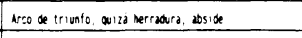 & $\therefore$ & 6.9 & & 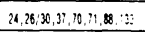 \\
\hline 5 & 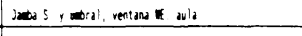 & $\therefore$ & 9 & & $33,70,11$ \\
\hline 16 & 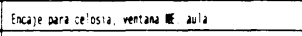 & $\therefore$ & 8 & & \\
\hline (1) & 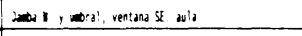 & be & 9 & & $x, 25,0,17,86,133$ \\
\hline 18 & 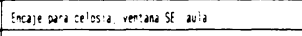 & $\therefore$ & 9 & & \\
\hline 19 & Detiost wid & is & 1.99 & & 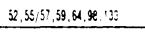 \\
\hline 26 & 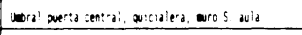 & $i$ & 3 & $+\infty e_{0}$ & $0,0,15,39$ \\
\hline 2. & 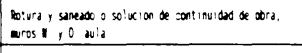 & 10 & $2,3,5,7,10$ & $1,8,9$ & \\
\hline 2. & 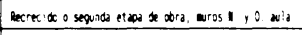 & $! 6$ & $2.3 .57,10$ & 1.8 .9 & $24.58,59,11,19,127$ \\
\hline [3] & 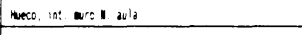 & It & 8 & 8 & 112 \\
\hline${ }^{24}$ & wreter a dunters & 1 & $3.6,7 / 8$ & $1,8,10,4.22$ & 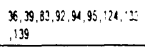 \\
\hline 25 & 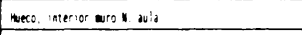 & $\therefore$ & 6 & $:$ & \\
\hline 26 & Imeress in: wrof wid & $\therefore$ & 9 & 8.4 & \\
\hline$z^{2}$ & 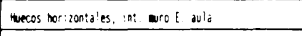 & $\therefore$ & 9 & 8.4 & 28 \\
\hline 36 & 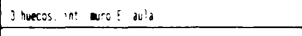 & . & 9 & 8,1 & 133 \\
\hline .9 & 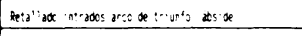 & $!$ & 6,9 & 8.14 & $133,14.199$ \\
\hline$x$ & Dos weres: :n: wro : wid & $\therefore$ & 9 & 0.4 .28 & 133 \\
\hline$y$ & 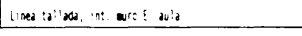 & $\therefore$ & 9 & 8 & \\
\hline
\end{tabular}

Fig.7.- Inicio del listado de elementos.

correlacionar con un período o estructura concreta, se colocaron en el listado entre los períodos a que más se acercaban por sus relaciones cronológicas relativas, con el auxilio de los signos < equivalente a "anterior a", $<<$ "anterior a elementos anteriores a" $\mathrm{y}>$ "posterior a". La agrupación de elementos en el listado bajo estos signos equivale a su situación relativa en la columna del diagrama ocupando un amplio tramo temporal o sin una colocación fija respecto a un período o estructura. Tampoco se efectuó un diagrama final de elementos, por la complejidad de relaciones dudosas que justamente estos elementos presentan. Sólo realizamos un diagrama final sintético con los elementos más representativos de cada período (Fig. 8). Creemos que con el listado y la planimetría, al menos teóricamente, cualquier investigador tiene los datos necesarios parapoder reconstruir nuestro procesode análisis, comprobándolo, reconstruyéndolo $\mathrm{y}$, en su caso, corrigiéndolo.

\section{La serie estratigráfica y estructural del edificio}

El primer periodo, prerrománico.-El análisis de paramentos permite conocer con todo detalle las características de este período. Su distribución topográfica, con restos en todos no estaba "cerrada", y que por ello no era posible $-$.

1. En el proceso de trabajo se mezclaron los términos etapa y período, equivalentes a fase. Período y fase pueden equivaler, pero etapa debe considerarse un subperíodo, por ejemplo "distintas etapas de obra de un período". 


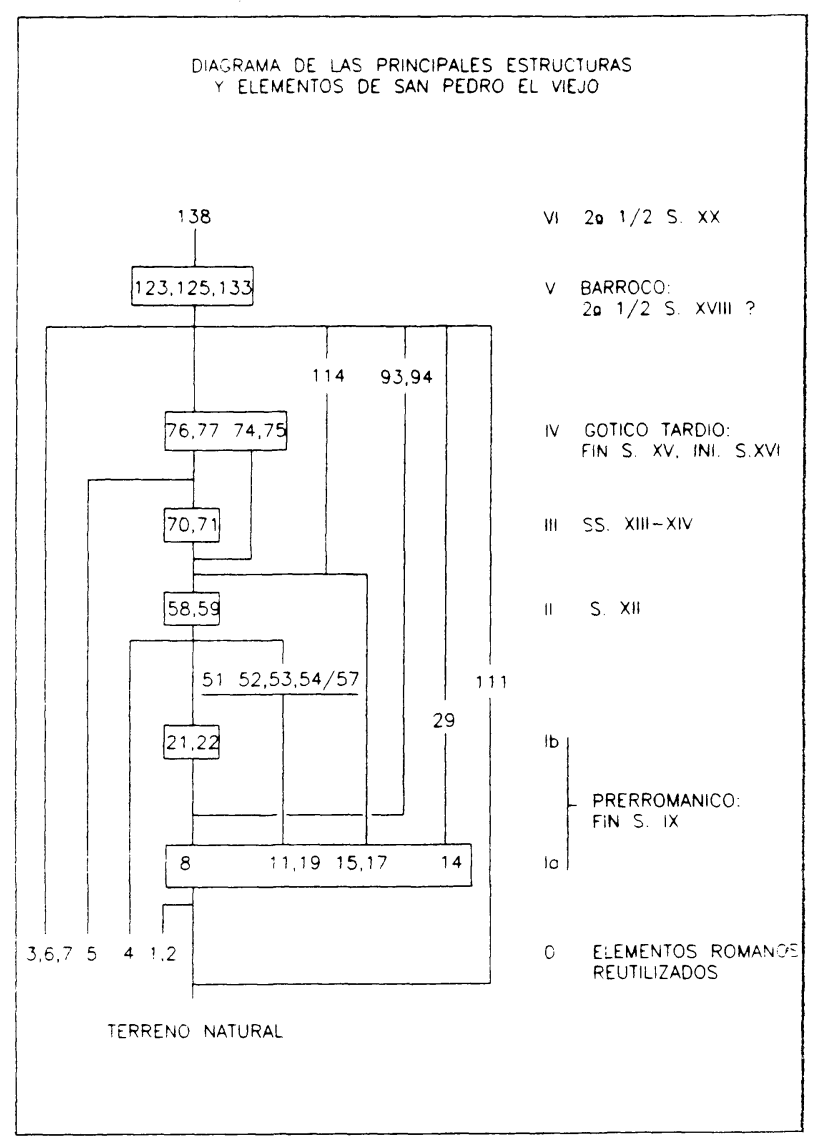

Fig.8.-Diagrama periodizado de los principales elementos, en cartuchos agupados por estructuras. los muros, a mayor altura en el muro $\mathrm{N}$. del aula y prácticamente entero el testero del aula y el ábside. Conocemos también su aparejo, formado por sillares de caliza, romanos, reutilizados y recortados como demuestran las inscripciones romanas -una en este período, las demás usadas de nuevo en momentos posteriores- y las abundantísimas huellas de gafas y grapas que presentan ( $\mathrm{E}$ $1,2)$; tallados una vez colocados en el muro, con guías de careado en sus aristas para sacar la cara de los paños exteriores, desbastados con azuela y puntero y alisados con cincel. Del mayor interés fue conocer que, en el interior del ábside y del testero $\mathrm{E}$. del aula, los sillares romanos no se retallaron, quedando comovenían del yacimiento expoliado que sirvió de cantera (Fig. 4). Es posible que escasos restos encontrados de un magnifico estuco (E 53,60) pertenecientes al edificio primitivo cubrieran estos sillares no alisados. La hilada inferior de cimiento era también de sillares romanos de arenisca gris azulada, resaltados y retallados hasta el nivel de la roca. Los muros laterales y el testero de pies del aula son de dos hojas que no atan entre sí -grosor $95 \mathrm{~cm}$-, mientras que el testero E. y los del ábside son de una sola hoja -grosor $47 \mathrm{~cm}$-; construidos de modo que las hojas interiores del aula y los muros del ábside apenas atan con el testero E. del aula.

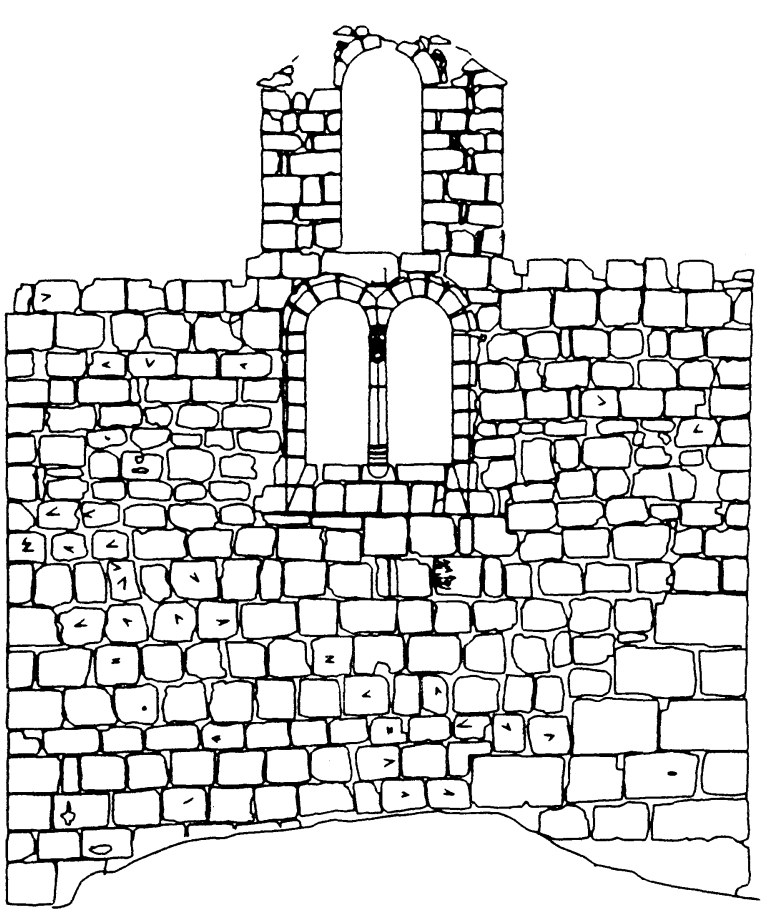

$A$

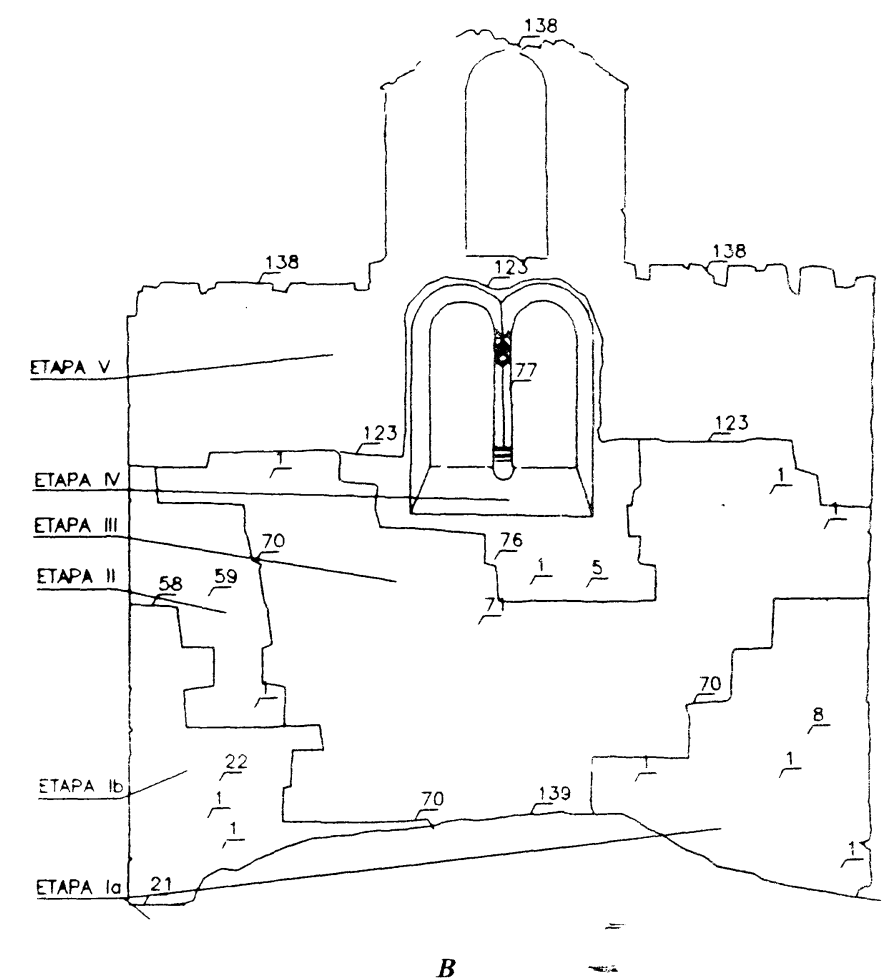

Fig.9.- Cara externa del testero occidental del aula. A, croquis de campo de la zona 6, utilizado para la diferenciación de los elementos. B, plano con la representación de las etapas. 
El aula tenía dos puertas en el muro S., posiblemente por estar dividida en dos zonas litúrgicamente diferenciadas; dos ventanas asaeteadas (E 15/18) y con molduras para celosías en el testero $E$. del aula, que indican que se dividía en tres naves; un arco de triunfo $(E 14,19)$ hoy retallado, que probablemente fue de herradura. El ábside tiene una ventana (E 13) ajimezada, de medio punto, también preparada para celosía; y su bóveda (E 12), construida con pequeños sillares de toba tallados in situ, apoyada en pechinas que nacen en arista de las cajas que dejan en las esquinas los tímpanos, por ser de diámetro menor que el ancho de las paredes, y cerradas en arco por encima de ellos, de modo que el arranque de la bóveda está volado y también su diámetro es menor que el ancho interior del ábside.

El problema fundamental de este primer período es el de justificar dos etapas distintas pero constructivamente muy similares, que hemos distinguido como Ia y $\mathrm{Ib}$ (E 8/24).

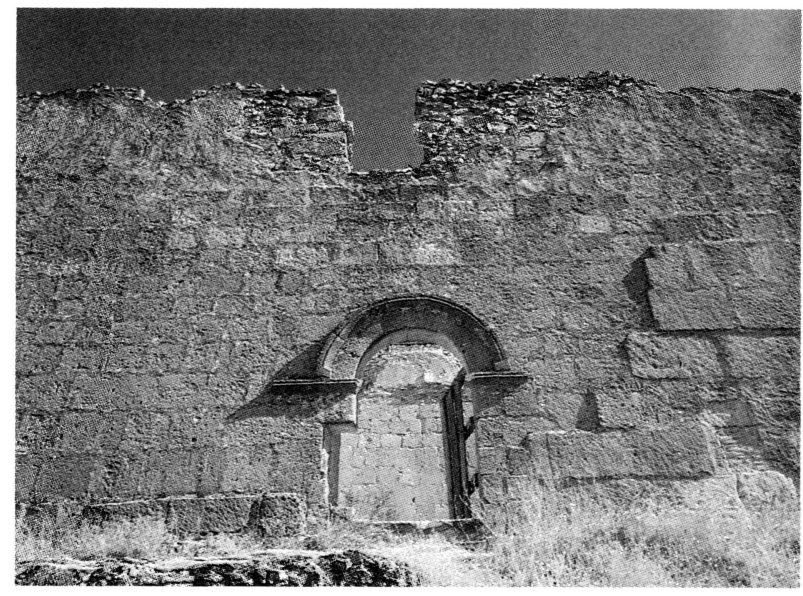

Fig.11.- Detalle del tercio central del muro $S$. del aula exterior. Se observa el corte debido a un derrumbe de la sillería alto medieval; la puerta tardo medieval encajada en el muro románico; y los dos recrecidos, el tardo medieval de sillería y el barroco de mampostería.
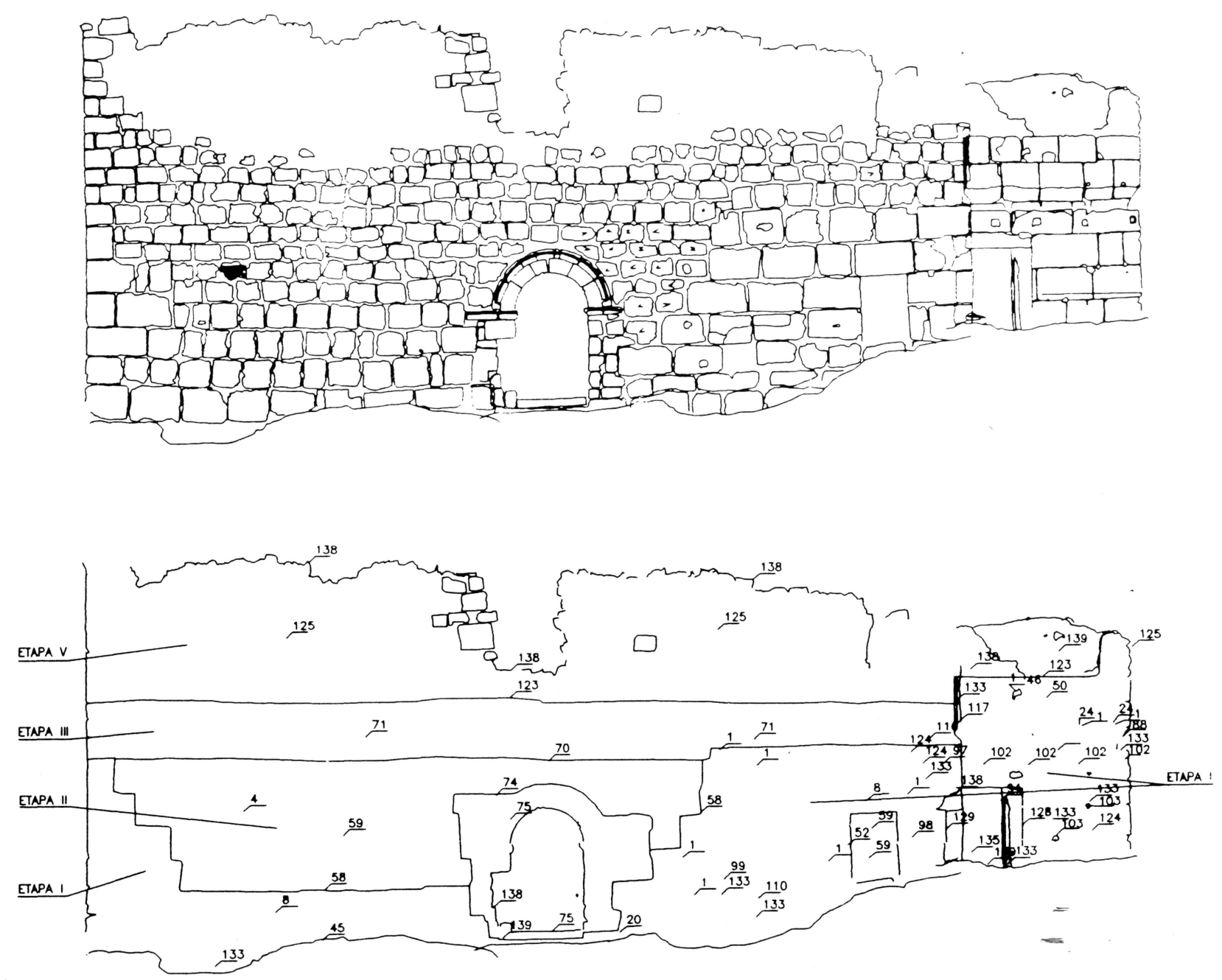

Fig.10 a.- Muro S., exterior. Restitución fotogramétrica y etapas. 


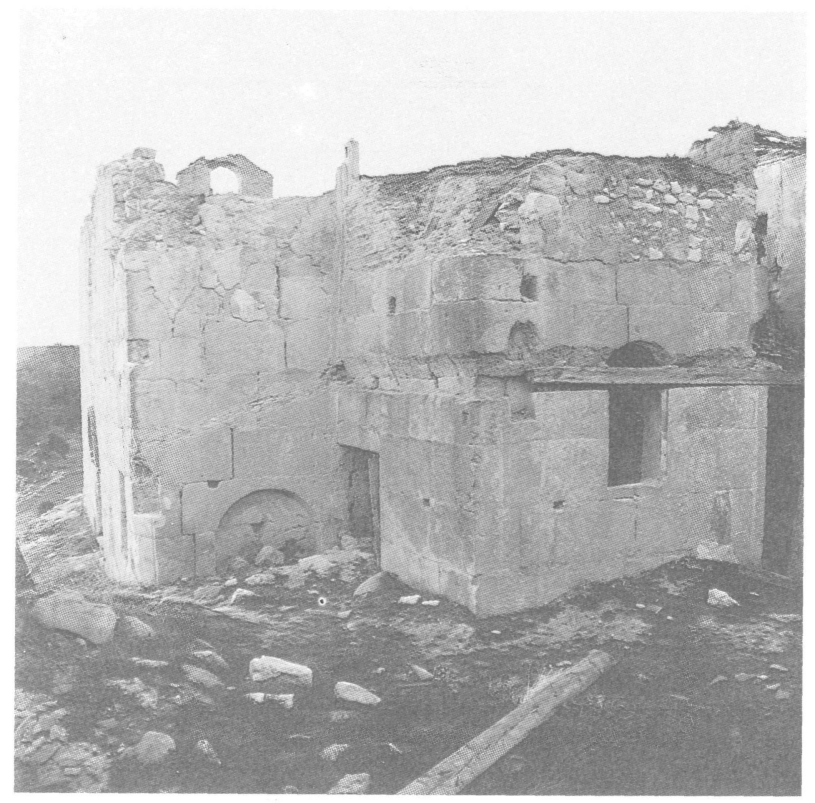

Fig.12.- Cabecera de S. Pedro el Viejo, desde SE.

En el muro N. y O. del aula es donde se diferencian, no sin dificultad, las dos etapas, por la presencia de mechinales para andamios en su etapa más antigua, por una superficie escalonada con talla en codo de sillares, que debe considerarse un corte de saneado, y por la diferencia de módulo de los sillares, mayores en la etapa más antigua altura 50/58; largo $40 / 88 \mathrm{~cm}$, llegando a sobrepasar el metro - y más pequeños y cuadrados en la segunda - altura 30/38; largo 39/52, llegando hasta $95 \mathrm{~cm}$-, quizás por haberse utilizado los sillares de la primera etapa recortándolos. De estos tres criterios el de los mechinales es el más seguro, dado que los otros pueden encontrarse aisladamente dentro de una misma etapa, como ocurre con juntas verticales y codos en la primera etapa de este mismo muro N., en su parte central. Toda la primera etapa del muro S. del aula, el testero oriental del aula y el ábside entero, y por lo tanto su cubierta abovedada, pertenecen a la etapa Ia por la presencia de mechinales.

Fue muy discutida la decisión de si estos dos estratos debían considerarse "etapas" de un mismo "período" o dos períodos $\mathrm{y}$, por consiguiente, dos estructuras distintas. Las similitudes constructivas entre ellos y la dificultad en diferenciarlos nos hacían pensar en un mismo período con dos etapas de obra, la inferior construida con sillares mayores; pero el criterio de presencia/ausencia de mechinales obligó a diferenciarlas optando por una solución intermedia: dos etapas separadas por una ruina (el corte E 21), que afectó al muro $N$. y la esquina NO. La ruina habría sido inmediata a la primera construcción, debido a sus defíciencias constructivas, muros de dos hojas, ausencia de enjarjes y quizás los empujes de la cubierta.
Las características y el funcionamiento de la primera construcción influyeron decisivamente en la historia posterior de la ermita. La cabecera siempre se mantuvo en pie, quizás gracias al efecto de atado que producía la bóveda, mientras que los muros laterales y trasero del aula tendían a colapsar, debilitados aún más por la puerta $\mathrm{S}$. y la ventana $\mathrm{O}$.

Periodo II, románico.- Entre los períodos I y II volvió a haber una ruina, que en el muro $S$. fue tan brutal que las hiladas que resistieron resbalaron significativamente de su lugar provocando escalonados (Fig. 11). El muro N. también resultó afectado por la restauración, aunque pudo tratarse más de una acción de saneado que propiamente de una ruina. La reconstrucción (E 59) reutilizó los sillares del período I, cortándolos, repasando sus caras con gradina y marcándolas con "V" y " $Z$ ". También se retallaron con gradina las caras internas del aula supervivientes de la estructura I, lo que indica que todas las caras interiores de la primera estructura tenían los sillares sin retallar, y que así siguieron los del testero E. del aula y el ábside, porque debían mantener su decoración de estuco. Debe admitirse que se sustituyó la puerta principal por una puerta nueva, a la que pertenecería una moldura de arco románica reutilizada en la actual, más tardía, y el descentramiento entre la puerta actual y el arco de descarga interior (E 62). Todo parece indicar que la ventana $\mathrm{O}$. del muro $\mathrm{N}$. se abrió ahora, indicio de la existencia ya en este período de un coro alto y de que el remate de los muros se situaba al menos dos hiladas por encima de la máximaaltura que han conservado los restos de este período. El espacio litúrgico del aula sufrió un cambio significativo al tapiarse la puerta delantera $\mathrm{y}$ construirse un coro alto.

Periodo III, gótico.-Una nueva ruina provocóla restauración del período III. Afectó severamente al testero O., donde debió destruir una parte conservada del período I, mientras que en el muro $\mathrm{S}$. se presenta -como ocurría en el período anterior en el muro N.- como un saneado perfectamente horizontal $(\mathrm{E} 70,71)$. La restauración vuelve a utilizar el mismo material romano de la iglesia prerrománica, ahora de tamaño más irregular y mezclado con sillares de arenisca de color ocre tostado. Su datación viene dada por su situación relativa en la columna estratigráfica y por la tipología de una lauda funeraria altomedieval incluida o reutilizada en el muro de los pies (E 68).

Período IV, renacentista.- El período IV parece deberse a una intención de mejora y enriquecimiento del edifício, colocándose nuevas puerta y ventana en los muros $\mathrm{S}$. y O. (E 74,76). La colocación de la ventana, decorada con los escudos del monasterio de Arlanza, se aprovechó para sanear parte del muro que debía tender de nuevo a abrirse siguiendoel esquema de las ruinas anteriores. Esta reforma coincide con la construcción de un edificio que engloba la cabecera y que cubre las necesidades de sacristíay vivienda. Estilísticamente responde a fines del s. XV ocomienzos del XVI. 

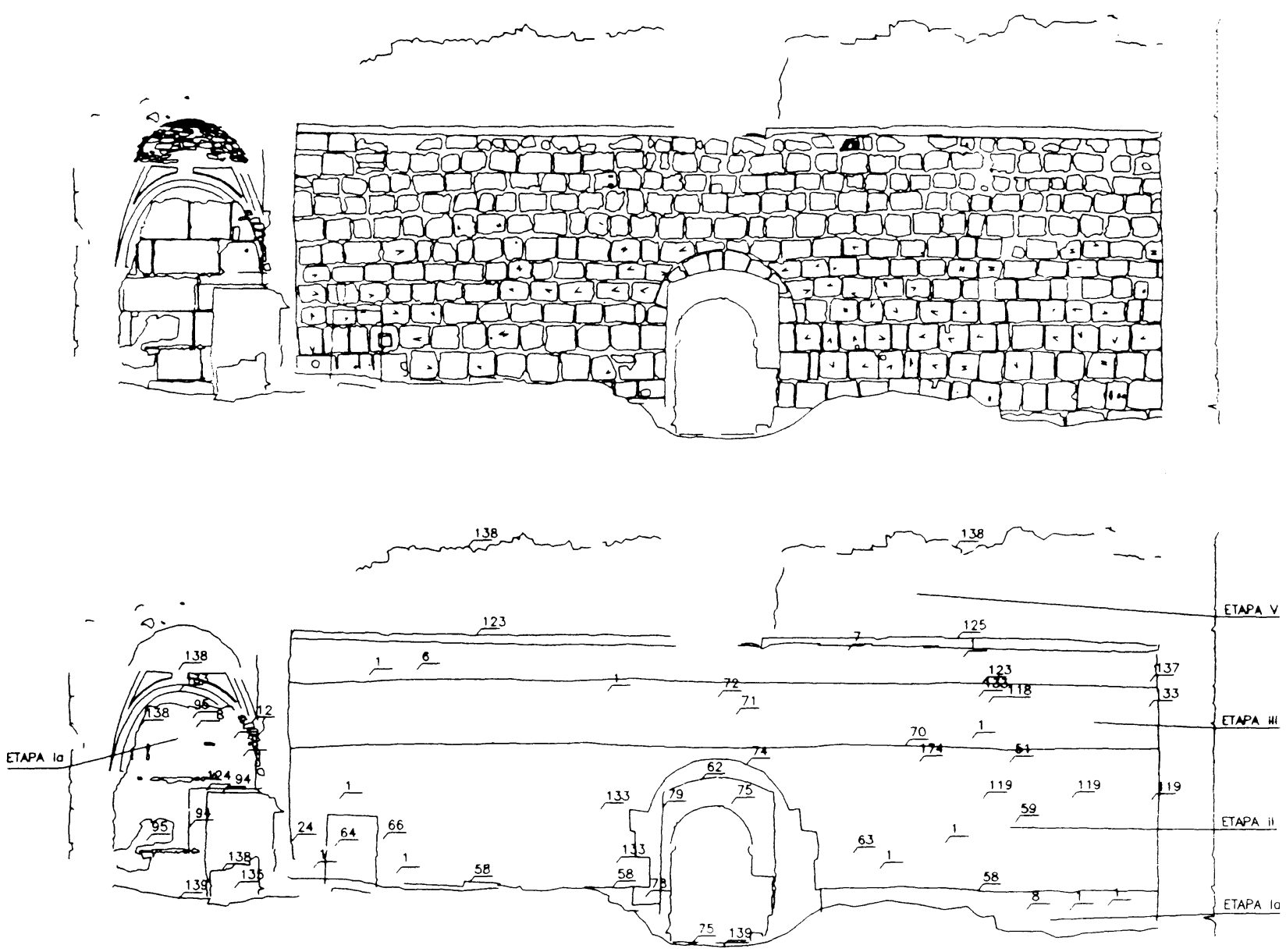

Fig.10 b.- Muro S., interior. Restitución fotogramétrica y etapas.

Entre este período y la obra barroca hay al menos dos subperíodos de difícil seriación, agrupados en el listado como $<<\mathrm{V} y<\mathrm{V}$. A ellos corresponden las intervenciones en el ábside de la apertura de sus puertas laterales (E 93, 94), el cierre de su ventana y el corte de la bóveda posiblemente para colocar un retablo $(E 87,88)$ y la apertura de un nicho en el exterior del testero oriental (E 121), además de diversos enlucidos y pinturas (E 95).

Periodo V, barroco.-El último período constructivo coincide con la expansión final del monasterio de Arlanza, que decide enriquecer la ermita siguiendo el modelo de la nueva iglesia que construye, entre 1751 y 1792 , Ventura Rodríguez para el cercano monasterio de Silos. Se sanearon $\mathrm{y}$ recrecieron todos los muros, utilizando adobe, sillarejo y mampostería, incluso los del ábside; se colocó la espadaña a los pies, se abrió una ventana encima de la puerta meridional y se cerró la románica del muro N., aunque se mantuvo el coro alto. Todo el interior de la ermita se estucó con pilastras y molduras planas, cubriéndose el aula con una bóveda de lunetos encamonada (E 123,125,133).

\section{Conclusiones}

Dados sus resultados, el análisis estratigráfico ha cumplido con las expectativas previas. No sólo conocemos la seriación constructiva de la ermita, con la "sorpresa" de descubrir que es el resultado de, al menos, cinco edificaciones consecutivas; sino también las características del primer edificio. Aunque del análisis no se deriva directamente una datación, sí se concluye un sistema de nuevos datos objetivizados que abre nuevas vías a su datación, y en última instancia plantea una datación más segura.

El mayor interés ha sido conocer en detalle el aparejo del primer edificio, con sillares romanos reutilizados cuyos frentes, una vez colocados, eran rebajados hasta sacar la cara del muro. La técnica es tan evidente y la morfología final tan similar a la supuesta sillería de época visigoda que ha permitido reconsiderar si otros ejemplos de ésta no serían también de sillería expoliada, como parece que efectivamente es, por ejemplo: en Quifitanilla, La Nave, Plá de Nadal o Marmelar, además de en S. Vicente del 
Valle, el pórtico del Valdediós, Sta. Coloma, Celanova, Lourosa, ...

La segunda aportación de la lectura ha sido la correlación de una serie de características, asignadas a la primera estructura y por tanto coetáneas al aparejo de sillería expoliada: ausencia de enjarjes en los muros, bóveda sobre pechinas, organización de la iglesia en tres naves y tres zonas litúrgicas (con el ábside), decoración estucada como en Sta. Coloma, tipos de ventanas, ...; serie que a su vez plantea paraestaiglesia un sistema reorganizado de paralelos con las demás iglesias altomedievales, donde se mezcla lo visigodo con lo omeya, lo asturiano y lo mozárabe. Un ejemplo prototípico es el de la bóveda y sus pechinas, en relación con la cercana Quintanilla, supuesta de época visigoda, con edificios sirios omeyas del s. VIII y con otros supuestos mozárabes del s. X de Álava, La Rioja y Burgos. De aquí se induce una hipótesis, diferente a la tradicional visigotista, que supone este edificio formando parte de un sistema con técnica constructiva de raíz islámica y datable en el s. IX.

La lectura plantea también problemas que hay que interpretar según las hipótesis científicas al uso, aunque quizás respondan a planteamientos por hoy no evidentes. Es el caso de la diferenciación de las "etapas" la y Ib que hay que considerar si son o no coetáneas. Un planteamiento secuencial del tiempo histórico obligaría a considerarlas consecutivas: por ejemplo, si la Ia es visigoda, la Ib de reconquista, según la hipótesis neovisigotista. Pero la propia argumentación de la lectura de paramentos hace suponer la inmediatez -casi "correlación"- de ambas en base a su similitud de caractereres.

Finalmente la comprensión conseguida por la lectura permite plantear una hipótesis sobre la forma perdida del edificio (Fig. 15). La ventanas del testero del aula, el grosor de sus muros laterales y la fuerza que provocó su ruina, hacen pensar que sus naves estuvieran abovedadas sobre arcadas de cinco vanos que correrían sobre pilares. El edificio parece medido en codos de aproximadamente 50 $\mathrm{cm}$ que ordenan el espacio en cuadrados de 5 unidades de lado, que sólo dejan fuera los muros de testero. A través el aula mide 15 codos que se distribuirían en tres grupos de 5: 5 para la nave central y 5 a cada lado, uno para la arcada, dos para la nave y dos para el muro. A la larga cada tramo equivaldría a 5 codos: cuatro para su vano y medio para cada medio pilar que le corresponde a cada lado. El interior del ábside equivale también a un cuadrado de $5 \times 5$, de modo que su ancho continúa el de la nave central y sus muros el grueso de las arcadas. Esta reconstrucción semeja proporciones asturianas, como, por ejemplo, las de Valdediós.

El método es útil en la consecución de relaciones isócronas -correlación de estructuras o de actividades- y sucesivas. Perola datación absoluta sigue en manos de otros métodos, como era de esperar. En nuestro caso, de la tipología de los elementos, que por hoy depende, a su vez, de los paradigmas aceptados previamente -visigoda si se sigue un paradigma visigotista; de "reconquista" si se acepta el paradigma islámico-, por lo tanto con un valor a su vez muy relativo. Pero es esta relatividad la que este método ayuda a superar con la aportación de nuevos datos como creemos haber demostrado con nuestro caso. Ello no impide que se complete con otros métodos, como debería hacerse aquí con la excavación arqueológica y la restauración monumental, complementaria la primera del conocimiento y necesaria la segunda para resolver la precaria situación de un documento histórico que está a punto de perderse.

\section{BIBLIOGRAFÍA}

ABÁSOlO, J. A. y GARCíA ROZAS, R., 1980: Carta arqueológica de la provincia de Burgos. Partido judicial de Salas de los Infantes, Burgos.

AMADOR DE LOS RÍOS, R., 1896: Las ruinas del monasterio de S. Pedro de Arlanza en la provincia de Burgos. Estudio histórico-arqueológico, Madrid.

ARBEITER, A., 1990: Die Westgotenzeitliche Kirche von Quintanilla de las Viñas. Kommentar zur Architektonischen Gestalt, Madrider Mitteilungen, 31, 393-427.

BROGIOLO, G. P., 1988: Archeologia del'edilizia storica, Como.

CABALLERO ZOREDA, L., 1991: Observaciones sobre materiales y tecnologías de construcción de época visigoda en España y Portugal, en Marini, L. (ed.), Materiali da costruzione $e$ techniche edili antiche. Indagini e rilievi nell'ottica della conservazione, Alinea editrici, Florencia, 25-30.

ID. (cood.), (1992): Sobre el análisis arqueológico de construcciones históricas. La experiencia de Santa Eulalia de Mérida, la torre de Hércules en La Coruña y $\mathrm{S}$. Pelayo de Arlanza, en Arqueologia del monumento, III Encuentros sobre Arqueología y Patrimonio de Salobreña, Ayuntamiento de Salobreña (en prensa)

ID., (1993): Algunos aspectos de cultura material en época visigoda y postvisigoda. A propósito de la datación de Santa María de Melque, El s. VIII. Islam y Occidente, un primer encuentro, Casa de Velázquez y Universidad de Alcalá de Henares, (en prensa).

ID. 1994: Un canal de transmisión de lo clásico en la alta Edad Media española. Arquitectura y escultura de influjo omeya en la Península Ibérica entre mediados del s. VIII e inicios del s. X, (I) y (II), Al Qantara, 15, 321-348 y 16,107-124.

ID., CÁMARA MUÑOZ, L., LATORRE GQNZÁLEZ-MORO, P. Y MATESANZ VERA,P., 1991/1992: La jiglesia prerrománica deS. Pedroel Viejo de Arlanza (Hortigüela, Burgos), Numantia,5, 139-165. 


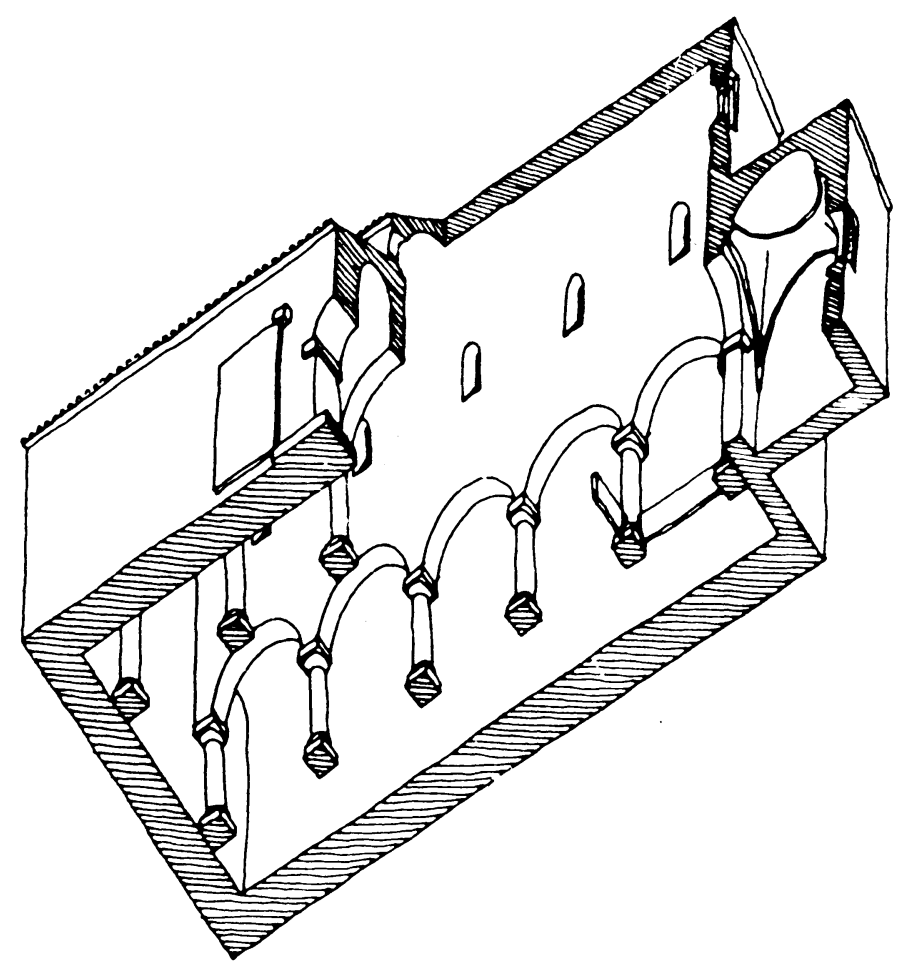

Fig.13.- Reconstrucción ideal del primer edificio.

CAMPS CAZORLA, E., 1939/40: El visigotismo de Quintanilla de las Viñas, Boletín del Seminario de Estudios de Arte y Arqueología, 22-24, 125-134.

CRESWELL, K.A.C., 1969: Early Muslim Architecture. I. Umayyads A.D. 622-750, Oxford.

FONTAINE, J., 1977: L'Art prerroman hispanique, 2. L'Art mozarabe, Zodiaque.

GÓMEZ-MORENO, M., 1966: Primicias del Arte cristiano español, Archivo Español de Arte, 39, 101-139.

HAMILTON, R. W., 1959: Khirbat al Mafjar: An Arabian Mansion in the Jordan Valley, Oxford.

HAUSCHILD, Th., 1972: Westgotische Quaderbauten des 7 Jahrhunderts auf der Iberischen Halbinsel, Madrider Mitteilungen, 13, 270-285.

HUIDOBRO, L., 1922/25-1926/29: El monasterio de S. Pedro de Arlanza y su primer compendio historial inédito, Boletín de la comisión provincial de monumentos históricos y artísticos de Burgos, 1 y 2, 199-207 y 211-214.
ID., 1926/29: El arte visigótico y de la reconquista en Castilla Nuevos descubrimientos, BCPMHA de Burgos, 2, 361-369 y 394-404.

MOREDA BLANCO, J. Y NUÑO GONZÁLEZ, J., 1987: Excavaciones en el monasterio de San Pedro de Arlanza, Hortigüela (Burgos), II Congreso de arqueología medieval española, 3, 557-569.

OSABA Y RUIZZ DE ERENCHUN, B., 1976: El arte árabe y sus huellas, en Arteburgalés. Quince mil años de expresión artistica, Vitoria, 68-83.

PARENTI, R., 1988: Le tecniche di documentazione per una lettura stratigrafica dell'elevato, en Francovich, R. y Parenti, R. (edit.), Archeologia e restauro dei monumenti, Consiglio Nazionale delle Ricerche. Università degli Studi di Siena, Florencia, 249-279.

SCHLUNK, H. y HAUSCHILD, Th., 1978: Die Denkmäler der frühchristlichen und westgotischen Zeit, Hispania Antiqua, Mainz am Rhein.

SERRANO, L., 1925: Cartulario de San Pedro de Arlanza, Madrid.

\section{Equipo Técnico}

Director: L. Caballero Zoreda, Centro de Estudios Históricos, CSIC.

Análisis arqueológico: L. Cámara Muñoz, arquitecto; P. Latorre González-Moro, arquitecto; P. Matesanz Vera, arqueólogo. Colaboradora: C. Sánchez Hernández.

Fotogrametría: G. Roibás e I. Merino Sepúlveda, ETSIA, Universidad Politécnica de Madrid. E. Ducar Martínez, estudio Latorrey Cámara. Los trabajos se realizaron entre 1991 y 1993 con permiso y subvención de la Junta de Castilla y León. Forman parte del proyecto de investigación "Sitio histórico", CICYT, PAT 89-0665. 